\title{
Intelligent Agents for Social and Learning Logistics Systems
}

\author{
Otthein Herzog (D) and Ingo J. Timm (D)
}

\begin{abstract}
The digitalization of logistics processes is often based on distributed models and decentralized control. As these logistics models constitute an important part of Industrie 4.0 concepts they must be powerful enough to cover dynamic processes and must enable a host of functions such as goal-oriented, reactive, pro-active, communicative, cooperative, competitive, and learning behaviors. In addition, these distributed models must allow for simulating, planning, allocating, scheduling, and optimizing logistics tasks. This implies that they must be able to act through communication channels with each other thus establishing logistics social communities.

Multiagent Systems (MAS) have been around for more than 30 years and lend themselves to the implementation of these distributed models needed for autonomous and cooperating logistics processes. It will be described and also demonstrated by three case studies why MAS are well suited for social and learning logistics systems. It will be shown how the resulting distributed MAS models provide the required functionalities for production and transportation logistics including the handling of dynamic local events as an essential feature for the successful planning, scheduling, optimizing, monitoring, and control of global logistics processes.
\end{abstract}

Ingo J. Timm was affiliated to LogDynamics until 2006 as Postdoctoral Researcher for Artificial Intelligence at the Center for Computing Technologies of the University of Bremen, Germany

O. Herzog $(\bowtie)$

Tongji University, Shanghai, People's Republic of China

University of Bremen, Bremen, Germany

e-mail: otthein.herzog@uni-bremen.de

I. J. Timm

SDS Branch Trier, Cognitive Social Simulation, German Research Center for Artificial Intelligence (DFKI), Trier, Germany

FB4 - Business Informatics I, Trier University, Trier, Germany

e-mail: ingo.timm@dfki.de 


\section{Introduction}

Logistics as an interdisciplinary field of research between engineering (mechanical engineering, production technology), natural sciences (mathematics, computer science), and economics has gained considerable importance since the early 1990s. At the beginning of LogDynamics in Bremen, logistics was strongly influenced by economic and political changes, i.e., reduction of manufacturing depth within an enterprise, concentration on core competences, global production, as well as liberalization of European's transportation market. Production and transportation processes are getting more complex, dependencies between processes in logistics networks and the global distributed Production are increasing, must be coordinated throughout complex networks bringing together various enterprises. In consequence, complex planning, coordination, and optimization problems challenge information systems in logistics since the 90s. This development is followed by the trend to digitalization, i.e., the dramatic increase of electronic business and electronic commerce as well as the development of ubiquitous and mobile computing and the Internet of Things. Nowadays almost any device, product, or process step can be supported or controlled by digital means. These developments are leading to new and innovative products and processes, e.g., based on small lot sizes, customer demand, flexible response, and customized mass production (mass customization). In its beginning, these developments have been addressed to by the Collaborative Research Center on Autonomous Logistics (SFB 637, funded by the $\mathrm{DFG}^{1}$ ) in Bremen, Germany. ${ }^{2}$

A holistic perspective on logistics is widely proposed, which considers both the business strategy and technical execution of automated systems. Scholz-Reiter et al. (2004), for example, introduce a 3-layer architecture for the specification and management of complex logistics processes, consisting of the decision layer, the information layer, and the execution layer (cf. Fig. 1a). These systems are each assigned to a specific disciplinary focus: Organization and Management (Decision System), Informatics Methods and Information and Communication Technologies (I\&C Technologies; Information System), as well as Material Flow and Logistics (Execution System). In the early days of logistics information systems, these three layers have been loosely coupled prohibiting situational or reactive behavior and decision-making, as the decision layer had no current information on the state or events in the execution system and computing resources in the information system where too limited for online (re-) planning. Developments of the Internet of Things are bridging the gap between information and execution system while agent technology mainly contributed to the information and decision system.

In context of Industrie 4.0, technological achievements should be used for realtime coupling of these three levels by bringing together Internet of Things and agent

\footnotetext{
${ }^{1}$ DFG: German Research Foundation (Deutsche Forschungsgemeinschaft)

${ }^{2}$ http://sfb637.uni-bremen.de/?\&L=2
} 

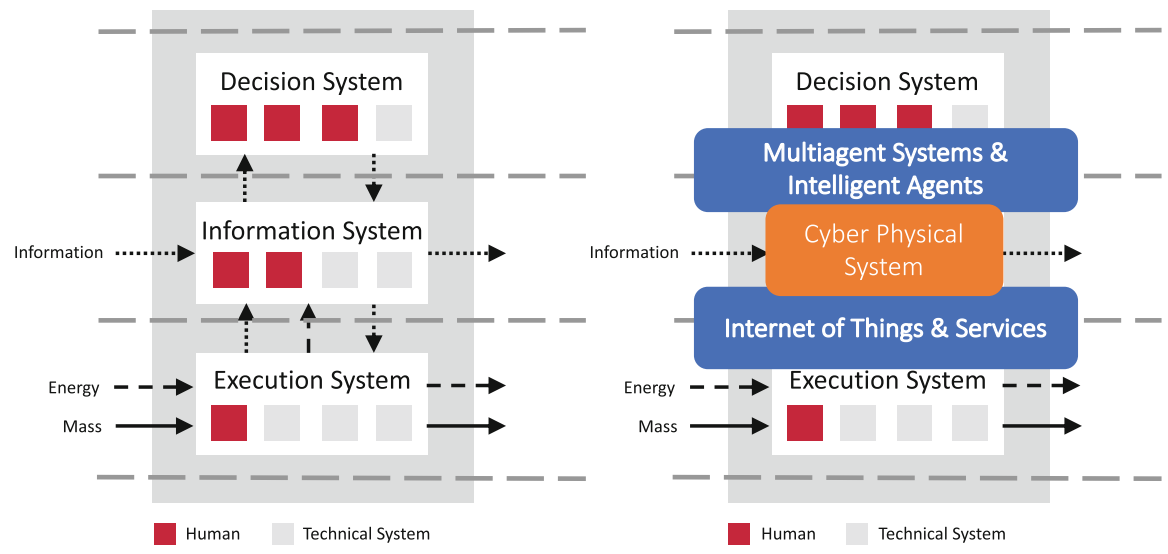

Fig. 1 Holistic perspective on logistics (a) with relevant technological innovations (b). adapted from (Scholz-Reiter et al. 2004)

technologies in the so-called Cyber Physical Systems (cf. Fig. 1b). Logistics systems are considered as autonomous (sub-) systems, where the behavior of individual actors depends on other actors or (sub-) systems in the "neighborhood." The autonomous systems interact with each other for pursuing their individual goals and the goals of the respective stakeholders. Thus, they are implementing local optimization, which leads to emergent behavior. As of this, it is uncertain how to guarantee, that individual behavior and emergent effects do not lead to global chaos, where no reliable prognosis on the system's outcome is feasible. Consequently, new challenges in modeling, engineering, and implementing autonomous logistics arise, which are related to behavior and management of groups in social sciences. In addition to the coordination problem of the 1990s, control and configuration of products and processes in production and logistics have also become increasingly relevant for future logistics information systems. To enable full potential of these technical and economic developments, future logistics information systems must handle knowledge about products and processes as well as their configuration or variation, additionally. As the requirements are shifting during runtime, future logistics information systems will require sophisticated social and learning abilities too.

In this chapter, we introduce intelligent software agents as promising technological solution for planning, designing, optimizing, and implementing social and learning logistics systems. We discuss innovative and application-oriented technological contributions from our research in LogDynamics on Intelligent Agents and modern logistics systems. Subsequently, we present case studies showing how Intelligent Agents implement logistics not only processes but outperformance conventional approaches. Complexity of logistics management is even of increasing complexity due to a wide variety of products and transportation processes, logistics networks, stakeholders, as well as domains. 


\section{Foundations of Multiagent Systems in Logistics}

In the late 1980s, Intelligent Agents and Multiagent Systems (MAS) have been invented as innovative software technology in the field of distributed artificial intelligence for implementing distributed systems. As they do not require fully standardized communication or interaction mechanisms, MAS were seen as a silver bullet for highly heterogeneous real-world systems like logistics. Heinz Jürgen Müller (1997) introduced an engineering method for modeling MAS and identified three main characteristics that an application domain requires for a fruitful application of MAS: "The application should show natural distributivity, e.g., autonomous entities, geographical distribution, distributed data; have a need for flexible interaction, e.g., there is no a priori assignment of tasks to actors, there are no fixed processes; be embedded in a dynamic environment, e.g. our physical world, artificial worlds like the internet, the world of finance" (Müller 1997, p. 218). With the AWIC-methodology, HJ Müller also defines the core elements to be specified when applying MAS: agents, world, interaction, and coordination. The active elements in the system to be modeled should be represented by agents, the realworld is abstracted, such that the actions of agents can be performed, and relevant information can be perceived in the represented world. Interaction of and between agents as well as coordination are important mechanisms for the behavior of the group of agents. In consequence, design and implementation of agent interaction is a core challenge for engineering autonomous systems balancing restrictive interaction for enabling reliable behavior and permissive interaction allowing for flexibility and emergent behavior (Krempels et al. 2006). Furthermore, interaction between agents can dynamically constitute organizational structures, as required in business applications, i.e., the organization of MAS emerges from interaction (Ferber 1999; Timm et al. 2006a).

Knirsch and Timm (1999) discuss the applicability of MAS to logistics with respect to Müller's characteristics natural distributivity, flexible interaction, and dynamic environment. Moreover, MAS has the potential to influence the organization and configuration of businesses models themselves, especially concerning inter-business transactions. So, with MAS even short-term cooperation, e.g., temporal logistics networks, becomes feasible from an information systems perspective, as "Agents do not only 'know' about the system's configuration but even 'notice' events occurring and react accordingly" (Knirsch and Timm 1999, p. 214).

However, MAS could not gain acceptance at that time because, on the one hand, theory and practice were far apart and there was a lack of standardization and efficient implementation. With the FIPA-Initiative (Foundation for Intelligent Physical Agents), the community introduced a platform for standardization of an abstract architecture, an agent communication language (FIPA-ACL) as well as a broad set of interaction protocols (Poslad and Charlton 2001). These standardization efforts were liberally accepted by the community enabling cross-border communication and coordination of agents and MAS from various developers and on multiple platforms. In the German Priority Research Program on Intelligent 
Software Agents and Business Applications (SPP 1083, funded by the DFG), the authors initiated a working group on bringing together multiple MAS in production logistics, developed at different research teams throughout Germany. With the Agent.Enterprise approach it has been shown that collaboration of heterogeneous MAS in logistics becomes feasible on the basis of the FIPA standardization (Stockheim et al. 2004). Finally, FIPA has been transferred to an IEEE standard in $2005 .^{3}$

In contrast to conventional software systems, Intelligent Agents and MAS should implement reactive and deliberative behavior, proactivity, and flexible interaction skills (Wooldridge and Jennings 1995). However, theoretical and methodological research mainly focuses on specific aspects of agent's and MAS' behavior leading to a clustered world of formalizations, reasoning approaches, and frameworks and tools. Therefore, Timm developed the Discourse Agent architecture which brings together the formal specifications of individual agents and MAS as well as the FIPA standardization (Timm 2004). The Discourse Agent specifies an architecture for agent behavior, knowledge representation, and inferences. It strictly separates internal and external behavior in a 3-layer-architecture, i.e., to separate communication with other agents (communicator level), interface to real-world entities or business software (executer level), and internal decision-making for reasoning on the agent's next actions (controller level).

Next to communication behavior and social abilities, autonomy is one of the main features for Intelligent Agents and MAS in logistics. For a better understanding and characterization of MAS approaches in logistics, Timm (2006) introduced levels of autonomy ranging from strong regulation to operational, tactical, and strategic autonomy (see Fig. 2).

Strongly regulated systems are conventional software systems, which implement deterministic system's behavior, i.e., routines and behaviors are defined in design time and there is no functionality within the software system to adapt its behavior to a new or unforeseen situation. Low-level learning or reactive behavior, which can be adapted by the agent itself leads to operational autonomy. In operational autonomy the agents adapt action parameters, e.g., low-level artificial neural networks in context of robotics, or choose between equivalent action steps with respect to the current situation. If the agent is also adapting the action sequences themselves or its planning behavior, it implements tactical autonomy. Here, adaptation takes place between different courses of action, e.g., structural adaptation of hidden Markov models. On a strategic level, an agent is capable of altering the goal or desire set itself or adapts the evaluation of goals and desires with respect to midterm experience.

\footnotetext{
${ }^{3}$ http://www.fipa.org/
} 

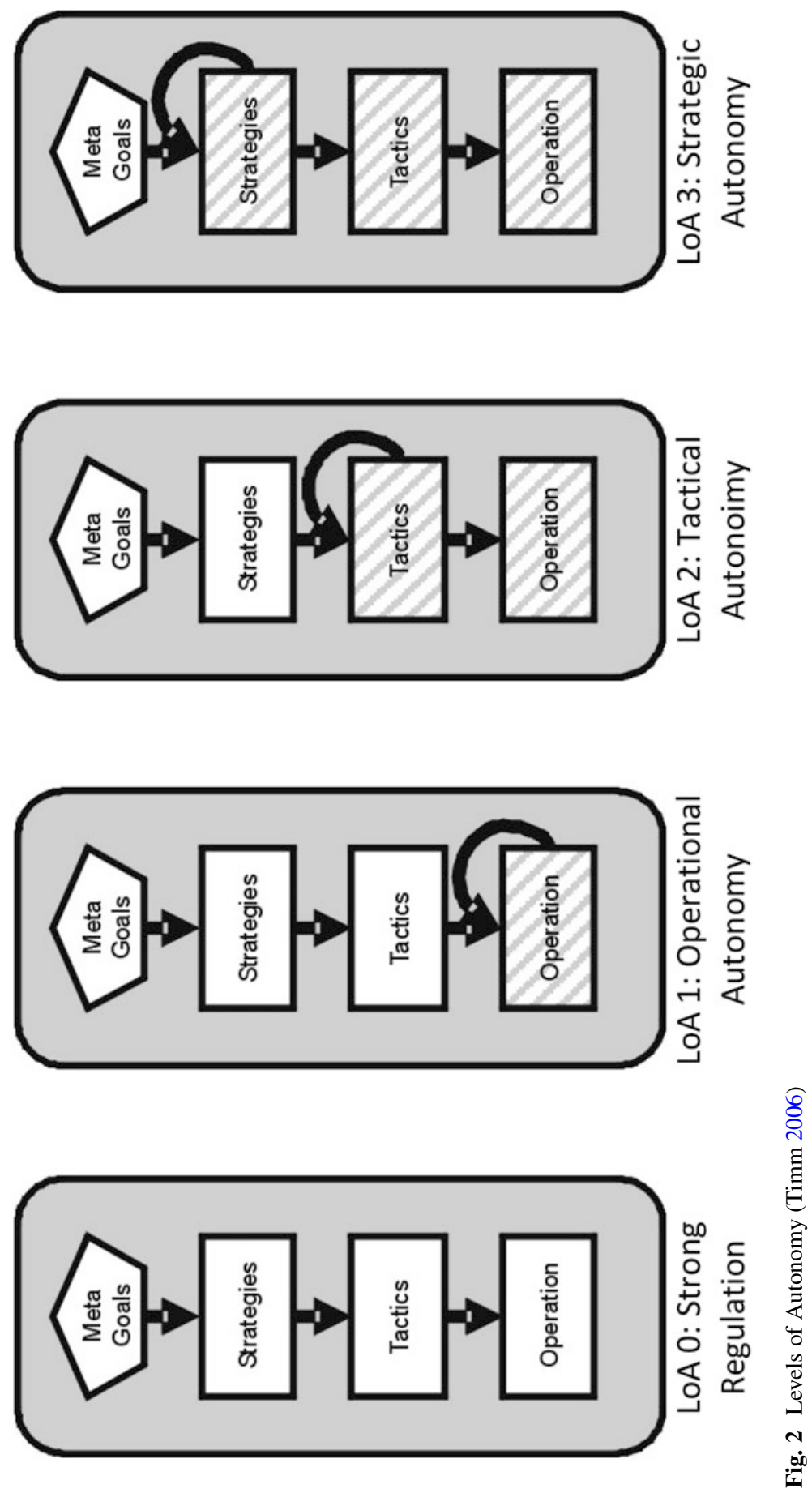


\section{Advanced Concepts for Multiagent Systems in Logistics}

A distinctive feature of MAS is the potential for collaboration among agents that depends, however, on advanced agent features such as internal knowledge management, the ability to learn, to plan, to negotiate, and to communicate effectively among themselves in order to reach a common goal. These functionalities require indepth knowledge about an application area (definitely not in a broad sense) and lead to autonomous MAS with potentially different operative roles, e.g., to select the best choice for the management of resources. The goal of such an agent society is rational and informed decision-making subject to their specific roles even if the partially visible environment of agents in an MAS increases the uncertainty in choosing the "right" decisions and the assessment of their utilities.

The most important prerequisite for informed decision-making is the ability to retrieve adequate knowledge for the assessment of situations that must be driven by utility, and also empirical knowledge for situation assessment (Gehrke and Wojtusiak 2008). This means that agents in an MAS must assume multiple roles: on the one hand, their specific domain-based roles, and, on the other hand, a knowledge management role where both producers and consumers are software agents. In order to achieve this, static domain knowledge must be available to the agents, e.g. a basic application domain ontology. In addition, empirical knowledge about the dynamics of the agent environment is needed that might be available through dedicated roles in order to enable situation assessment and prediction based on different knowledge models for decision-making involving decision, prediction, and classification models. Gehrke (2011) went a step further in researching how "relevant knowledge" could be secured by agents thus aiming directly at the semantic level and balanced decisions. A relevance-based information acquisition enables agents to identify, evaluate, and acquire exactly the information in a goaloriented and efficient way that have the highest relevance for an impending decision. As there could be a large number of possibly relevant information pieces, Gehrke (2011) succeeded in developing a method to reduce the relevance assessment to a minimal number of information elements using especially the d-separation of nodes in decision networks as a criterion for the stochastic impact of information on the expected action utility. In this way, it was possible to exclude irrelevant and information of lesser value from further consideration.

Warden (2019) selected a machine learning and knowledge management approach for information retrieval in that he developed a comprehensive methodology comprising a set of knowledge management functions for individual information retrieval and knowledge creation. He focuses on knowledge creation through induction of classification models that enables some flexibility with regard to applicable knowledge creation. The ability for induced models constitutes a competitive advantage for a learning agent by incorporating relevant knowledge that is distributed among an agent community of practice to improve and shape individual induction. This enables a wide range of knowledge-based decision support systems where it is not necessary from the beginning on to define the 
complete knowledge necessary for an application. Instead, in a society of learning agents, it is possible for an agent "to ask around" for knowledge necessary for the next decision, e.g. some classification knowledge. In detail, it could be shown that multiagent interactive adaptation of individual classification models by a communication process between advisor and advisee agents can lead to knowledge creation for advisees. From the machine learning perspective, it was demonstrated that the technique of argument-based machine learning could be transferred from an agent-human setting into a setup of an agent society. As far as the technology has been evaluated, it could be confirmed that the modular architecture of a flexible integration of heterogeneous induction techniques by different machine learning frameworks was instrumental for these results.

While the agents in Warden (2019) communicated without any presupposition about the reaction of their counterparts, the work of Luhmann $(1984,1995)$ provides guidance on how to take also the "double contingency" into account that is supposed to build a foundation of a sociality in its way how communication partners make use of slight indications of mutual dependencies. Therefore, it is possible that a history about the communication can be created that leads to self-accelerating dynamics of social order generation. In this way, also a dynamic social order between agents in a multiagent setting can be established. Berndt (2015) could show that Self-Organizing Multiagent Negotiations allow for both competition and cooperation among concurrently acting agents using iterated negotiations as a basic interaction method where multiple agents compete for their individually best outcomes. However, by creating groups of agents with the same objective, there can be cooperation within such a group paired with competition with other agent groups which is limited because the standard negotiation methods among concurrently acting agents are not guaranteed to achieve efficient coordination results because they are not aware of the existence of their agent counterparts, their capabilities, their objectives, nor their current activities.

This leads to the observation that the participating agents are not able to decide which actions to select to achieve the coordination results.

Berndt (2015) could show that the method inspired by the Luhmann (1984, 1995) observations, the Self-Organizing Multiagent Negotiations generate social structures among the negotiating agents making use of adaptive coordination methods. These structures reduce an agent's decision-making contingency by making the results of possible actions expectable as its expectations are driven by its interactions and the agent will be able then to select its own actions according to the estimated responses by other agents. In this way, the agents can explore possible results of their potential actions and thus overcome lacking knowledge. The resulting selforganization avoids mutual disturbances of concurrent activities and allows the contributing agents to achieve their objectives by stabilizing their interactions. This anytime coordination algorithm can approximate a Nash equilibrium, and it was proven by Berndt (2015) that the asymptotic effort of Self-Organizing Multiagent Negotiations could be reduced by the goal-directed activity selections to $\mathrm{O}\left(\mathrm{n}^{2}\right)$ in the number of all participants in contrast to conventional negotiations with an 
asymptotic effort of $\mathrm{O}\left(\mathrm{n}^{3}\right)$ at the expense of some additional memory requirements. The asymptotic computational effort could be shown to be $\mathrm{O}(\mathrm{n} \log \mathrm{n})$.

This social order generation process can be used to maximize the result quality even if the interaction and computation effort have to be limited, as it does not depend on the agent population size. A result quality evaluation could show that the capability to create a social order is crucial for multiagent teams or for the combinatorial coordination of multiple resources by a single individual agent and drastically outperforms conventional coordination methods. Using Self-Organizing Negotiations agent teams could be shown to outperform conventionally negotiating ones by up to $130 \%$ in their achieved welfare. This result is also well reflected in a real-world example where the Self-Organizing Multiagent Negotiations was applied to container logistics using sizable real-world data with almost 10.000 containers: the combined transportation and storage cost could be improved by almost 7\%.

The results presented in this section underline the high potential of MAS in logistics. However, there is a great challenge in engineering specific systems by distributing roles and capabilities (Ferber 1999): Should every agent be capable and responsible for any tasks, or should each agent be specialized on one role only? The resulting problem is to dynamically balance efficiency (specialization) and reliability (generalization) with respect to current workload situations. Optimal solutions may be obtained by learning from human cooperative systems using psychologic research. Timm et al. analyzed intentional forgetting as an interdisciplinary concept to adapt human and AI-teams in the German Priority Research Program on Intentional Forgetting in Organizations (SPP 1921, funded by the DFG) (Timm et al. 2019). On this basis, an MAS extension for situational adaptation of processes and roles by learning and forgetting of actions, plans, and desires has been developed (Reuter et al. 2020; Timm et al. 2020). This leads to a new understanding of engineering and situational balancing specialization and generalization in logistics. Moreover, this approach can be used for hybrid teams, i.e., human-AI-workforces and has great potential for qualification planning of human teams and capability selection for MAS.

\section{Case Studies}

In this section, we present three case studies showing the potential of social and learning MAS in logistics. The first case study is dealing with production logistics. In manufacturing, efficiency and effectivity are often lost in process planning by fixing details on required production methods or the selection of resource types. Such pre-determinations prevent flexible reaction even of MAS to specific situations or events. In the manufacturing domain, the authors have developed an approach for integrated process planning and production control in a real-world scenario. On a larger scale, logistic networks can significantly benefit from the flexibility of MAS as shown in the SPP 1083 (Kirn et al. 2006). However, a more general 
approach is required, i.e., in the SFB 637, the concept of autonomous logistic entities has been developed. As in Industrie 4.0, such autonomous decision entities can be implemented using MAS. In various real-world applications, MAS have been proven to be beneficial in contrast to conventional approaches. Such applications and results are presented in the second case study. The third case study is addressed to the conceptualization of intelligent carriers, i.e., the intelligent container, which has also been developed within the SFB 637.

\subsection{Case Study 1: MAS for Production Logistics}

In the early days of production planning and process control, production and its manufacturing processes were primarily treated as coordination problems, i.e., processes were based on Tayloristic separation of preparatory activities (process planning) and implementation activities (production control) (Toenshoff 1999). The process planning was performed as a preparatory activity within or after product design. Production planning and control systems were restricted to optimize availability of material and resources as well as to allocate resources for largescale or mass production. Except for the bill of materials, almost no information about the product itself was required in production planning. However, since the 1990s, the requirements to production logistics changed significantly due to trends like customized and personalized products, small lot sizes, or changing customer demand. For improved flexibility, pre-determination in preparatory steps like process planning should be reduced and the production planning and control system should be based on knowledge on the (customized/individualized) product, and on the skills and capabilities of the resources too.

In the IntaPS-projects (2000-2006) as part of the Priority Research Programme (SPP 1083) on Intelligent Agents and Business Applications, the authors developed an integrated approach to process planning and production control. The IntaPS approach consists of two components: an MAS implementing an electronic market as a decentralized planning component and formal representations of manufacturing features and skills and capabilities of resources. Agents are representing production orders or resources, like machine tools which are both representing digital twins of the real-world orders and resources responsible for negotiating resource allocation and scheduling production in a decentralized manner. For the technical implementation of such a system, the challenge arises, that there is almost no pragmatic architecture for implementing Intelligent Agents in such an environment. Many architectures developed for Intelligent Agents are based on unrealistic assumptions as consistency of goals. In logistics, it is well known that many standard objectives are competitive, e.g. maximizing utilization leads to high work-in-process and longer lead times.

Thus, Timm (2004) formally defined and specified a reference architecture, the so-called Discourse Agents. In contrast to other approaches, this architecture is based on potentially conflicting goals within an agent and between agents. The 
internal conflicts are handled by cobac (conflict-based agent control), which is an extension to the well-known belief-desires-intention architecture (Rao and Georgeff 1995; Wooldridge 2000). The cobac approach is based on a logical representation of goals and desires within an intelligent agent. As a first step in the deliberation cycle, an agent identifies accessible desires on basis of the current situation. In the next step, the desires are compared and evaluated due to relevance, chance, and risk of pursuing or ignoring available objectives. The desires are then processed pairwise, the potential for conflict or synergy is calculated, and a conflict resolution strategy is applied accordingly, e.g., merging desires, removing one of the two desires, etc. Additionally, Discourse Agents allow for dynamic adaptation and learning in negotiations using Markov chains with the OAC algorithm (2004). Both algorithms have been widely evaluated using agent-based simulation and an approach for testing autonomous systems has been developed (Timm et al. 2006b; Timm and Schumann 2009).

However, the strong focus on individual autonomy in the IntaPS project and the Discourse Agents led to limited efficiency on the shop floor level. Therefore, the question arises how the agents can consider objectives on the team level, e.g., of the shop floor at a whole or the enterprise, without limiting autonomy significantly. In an interdisciplinary cooperation, Timm and Hillebrandt (2006) have developed a framework for reflection in MAS inspired by Luhmann's theory of group dynamics. The underlying idea here is that the agents act autonomously as long as global goals are not in danger. If global goals are expected to be missed, the different levels of autonomy will be restricted: first operational, then tactical, and finally strategic autonomy. Schumann et al. (2008) applied parts of this framework as "regulated autonomy" to the domain of job shop scheduling and evaluated the concept with respect to cost of control (switching between autonomous and central decisionmaking) and efficiency (lead time). The results show the feasibility as well as the efficiency of this approach.

In the IntaPS project as well as in the entire SPP 1083, it has been shown that Intelligent Agents and MAS are beneficial to incorporate flexibility in realworld applications as well as to coordinate logistics effectively (Kirn et al. 2006). However, in economics gain of flexibility is accompanied by loss of control on the strategic management level. Thus, a contradiction arises between strategic control of the logistics system and autonomous behavior of (sub-) systems (Dembski and Timm 2005). Consequently, additional measures must be taken to reach beneficial emergent behavior in autonomous logistics processes.

\subsection{Case Study 2: MAS-Based Autonomous Logistics Processes}

Besides the knowledge acquisition processes in MAS, a second important research area is how the planning, control, and monitoring of processes can be delegated to autonomous and decentralized agents in an MAS as the digital representatives of objects themselves. This approach is taken for the development of process 
control in Industrie 4.0 environments where supply network management becomes increasingly complex, dynamic, and distributed. The paradigm of autonomous logistics aims at automating process control by delegating decision-making to the participating logistics objects. Based on objectives imposed by their owners, these autonomous logistics entities can themselves plan and schedule their way through logistics networks. Each entity incorporates only its own parameters as well as those of cooperating entities. Therefore, the computational complexity can be reduced significantly and dynamics can be dealt with locally. Schuldt (2011) showed that agents in an MAS are well-suited to the implementation of decision-making by local data processing as well as the coordination of the individual autonomous logistics entities and therefore enable the application to real-world control of logistics processes where both logistics service providers and consumers can be modeled with intelligent agents. Schuldt (2011) developed specific interaction schemes that are required for autonomous logistics, e.g., interaction protocols for team formation of autonomous logistics entities that constitutes the foundation for jointly coordinating the primary logistics functions transport, handling, storage, and picking. In addition, interaction schemes for plan formation and team action were developed to satisfy complex logistics objectives.

Schuldt (2011) validated this novel approach based on real-world scheduling processes based on the procurement logistics processes of a major European retailer of consumer products with more than 1200 own shops and over 56,000 outlets in total. Previously manually controlled processes for the dispatching of over 11,500 shipping containers within one year were implemented MAS-based with autonomous control and validated with MAS-based simulation. The results show that automated process control for standard cases with autonomous logistics is applicable to satisfy even the challenging logistics demands of this retailer through allocating logistics resources efficiently and reliably. This approach even exceeds the efficiency of the manual approach: the MAS simulation showed savings of 2.6 million pallet-days in the warehouses per year by better utilizing free times at the container terminals.

Gath (2015) investigates how autonomous control can be implemented into current logistics processes for intelligent transport logistics, and which communication and negotiation mechanisms allow for MAS-based autonomous control in transportation logistics. He also specializes in the decision-making processes of autonomously acting agents to satisfy the dynamic requirements of transport service providers and their customers in order to identify the optimization potential of current transport processes in real-time situations.

In this context, Gath (2015) provides a framework to meet the advanced requirements for logistics transportation processes on complexity, dynamics, and customization, concentrating on stable communication and negotiation protocols for the synchronization in highly parallelized negotiations. In order to satisfy the real-time requirements, efficient routing algorithms were developed, which enable the agents to make optimal and also near-to-optimal decisions within a limited time frame where the concept of nested Monte Carlo search with policy adaptation was shown to be superior to a classical branch-and-bound approach to provide near- 
to-optimal solutions for routing problems. Through extensive evaluations of the agents' decision-making algorithms through established benchmarks, real-world groupage traffic, and real-world Courier, Express, and Parcel services (CEP), it could be shown that the developed algorithms outperform previous approaches. In contrast to full truckload traffic, in groupage traffic the complexity of process planning is even increased by highly volatile order situations, changing individual shipment qualities, and delivery/pickup time windows and further impeded by dynamics originating from unexpected events. The evaluation with real-world data provided by logistics enterprises show that multiagent-based autonomous control meets the sophisticated requirements of groupage traffic and CEP requirements and outperforms commercial dispatching software.

Icarte (2021a, b) extended this approach to the scheduling of equipment in openpit mines that excel through ambitious production plans making use of expensive machinery equipment despite frequent dynamic events hampering the execution of the plans. He could show with real-world open-pit-mine data that his scheduling method implementing digital twins of the equipment by a decentralized MASbased approach and emphasizing highly concurrent negotiations between the agents outperformed the current methods in respect to productivity, fleet size, and cost.

\subsection{Case Study 3: Multiagent Systems and the IoT: The Intelligent Container}

Hribernik et al. (2010) present a potential solution to integrate the information and material flows of autonomous cooperating logistics processes with a standardsbased approach. An MAS models the information flow of autonomous logistics processes where the material flow is also represented using the appropriate logistics sensor data (e.g., location, speed, stops, temperature) and integrated with the information flow. Opening the decision horizon of an MAS in such a way allows for much more sophisticated information processing, inter-agent communication, and better decision-making. The proposed concepts for the connection of the information and material flows are based on the EPCglobal Standard Framework Architecture (EPCglobal 2007, 2008a, b, 2009).

An application scenario is shown in Fig. 3: There are different entity connection types that are part of the material flow, the communicating agents as its digital twins, and the relations among material and information flow. The integrative solution concept is based on four solutions to represent these relationships: (1) EPC for the unique identification of physical and virtual objects, (2) EPC Information Services as a gateway between MAS and the logistics objects in the material flow (see Fig. 3), (3) standard EPCIS events model the relations between the entities where adequate or proposed extensions to standard event types or new event types. (4) The integration of sensor data is achieved by an extension to the ALE (Application Level Events) standard. The application and extension of the widely adopted EPCglobal 


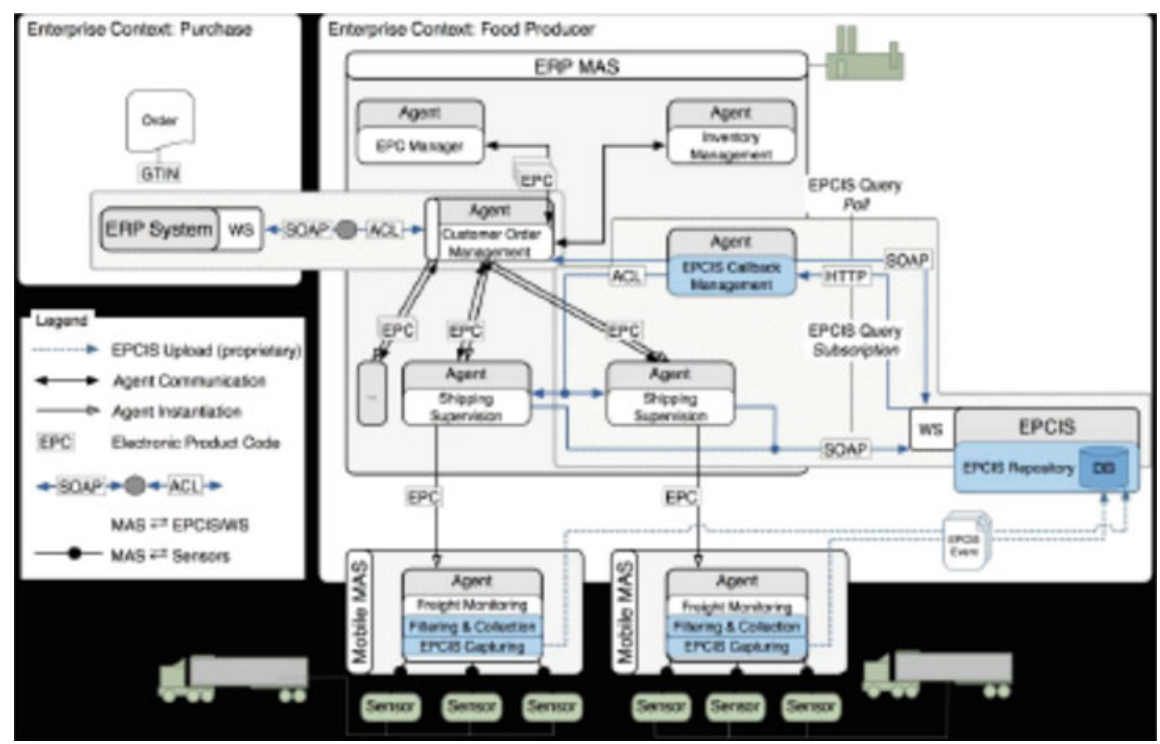

Fig. 3 Concept for an EPCIS-enabled implementation of the MAS outlined in the application scenario (Source: Hribernik et al. 2010, p. 60)

Architecture Framework to the problem allows for a standards-based solution that provides a platform-independent interoperability.

Dittmer et al. (2012) point out that the IoT focuses on technologies for data collection and distribution including standardization aspects such as EPCglobal (EPCglobal 2007, 2008a, b, 2009). However, this covers only the macro level of logistics systems whereas distributed logistics systems are designed for decentralized decision-making based on agent communication. Therefore, the approach of Autonomous Cooperating Agents defines the micro level of the logistic objects' behavior complementing the macro level. In the case of the Intelligent Container (Jedermann et al. 2007; Lang et al. 2014) this has been demonstrated by an implemented system for monitoring the ripeness of bananas in containers during their transportation from South America to Europe: here the Integrated Container can provide information about the shelf life of its cargo to the logistics network. To estimate the remaining shelf life from the data, decision support algorithms must be implemented based on the temperature history for the estimation of the changes of the fruits. The initial status of the fruits is determined by a visual analysis system of their color before they are loaded into a container. The container itself is equipped with a wireless sensor network that monitors the internal container status and also itself for the management of the sensor nodes and for failure detection. In addition, a decision algorithm has been developed that runs on sensor nodes and determines the temperature related quality losses, measurement intervals, sensor self-tests, and a sensor for the ripening indicator ethylene. Using the collected data during the transportation, a decision algorithm runs on sensor nodes that determines the remaining shelf life of the fruits. In this way, the Intelligent Container implements the new logistic paradigm of dynamic FEFO (First Expire First Out) 
as the remaining life time of the transported fruits is used to control the logistics process.

\section{Discussion and Perspectives}

It could be shown that the Distributed Artificial Intelligence technologies of Intelligent Agents and MAS is very well suited to build comprehensive models for autonomous cooperating logistics processes. This application area is characterized by highly uncertain events and processes where it is essential to rely on local resolutions that are worked out through the social interaction of the locally affected agents. In this way, decisions can be taken by design at the latest possible point in time, thus enabling the best possible outcome in a robust, resilient setting. In addition, learning (and forgetting) MAS models of logistics systems is able to preempt future adverse developments and thereby improve the local decisionmaking. The Collaborative Research Center on Autonomous Logistics (SFB 637) at the University of Bremen could demonstrate with its numerous publications and also by a substantial amount of successful SFB 637 start-ups that this route to the digitalization of logistics processes is a valid one and is being taken up by the logistics industry. The need for this kind of logistics digitalization became obvious during the first months of the COVID-19 pandemic where non-digitalized logistics networks had an almost complete breakdown, whereas digitalized logistics networks could recover within a comparably short time frame.

\section{References}

Berndt, J.O.: Self-Organizing Multiagent Negotiations Cooperation and Competition of Concurrently Acting Agents with Limited Knowledge. Doctoral Dissertation, University of Bremen (2015)

Dembski, N., Timm, I.J.: Contradictions between strategic management and operational decisionmaking - impacts of autonomous processes to decision-making in logistics. In: Palwar, K.S. et al. (eds.): Innovations in Global Supply Chain Networks. Proceedings of the 10th International Symposium on Logistics (ISL 2005), Lissabon, pp. 650-655 (2005)

Dittmer, P., Veigt, M., Scholz-Reiter, B., Heidmann, N., Steffen, P.: The intelligent container as a part of the internet of things. In: Proceedings of the 2012 IEEE International Conference on Cyber Technology in Automation, Control and Intelligent Systems, pp. 209-214. IEEE, Piscataway (2012)

EPCglobal Inc: EPC Information Services (EPCIS) Version 1.0.1 Specification. EPCglobal Inc., Standard Specification, New Jersey (2007)

EPCglobal Inc: EPCglobal Object Name Service (ONS) 1.0.1. EPCglobal Inc., Standard Specification, New Jersey (2008a)

EPCglobal Inc: EPC Tag Data Standards Version 1.4. Standard Specification, New Jersey, EPCGlobal Inc (2008b)

EPCglobal Inc: The EPCglobal Architecture Framework, 1.3, Standard Specification. EPCGlobal Inc, New Jersey (2009) 
Ferber, J.: Multi-agent systems: An Introduction to Distributed Artificial Intelligence. AddisonWesley, Boston, MA (1999)

Gath, M.: Optimizing Transport Logistics Processes with Multiagent-based Planning and Control. Doctoral Dissertation, University of Bremen (2015)

Gehrke, J.D.:Relevanzbasierte Informationsbeschaffung für die informierte Entscheidungsfindung intelligenter Agenten. Doctoral Dissertation, University of Bremen (2011)

Gehrke, J.D., Wojtusiak, J.: Traffic prediction for agent route planning. In: Bubak, M., van Albada, G.D., Dongarra, J., Sloot, P.M.A. (eds.) 8th International Conference on Computational Science 2008, vol. 3, pp. 692-701. Springer, Kraków, Poland (2008)

Hribernik, K., Warden, T., Thoben, K.-D., Herzog, O.: An internet of things for transport logistics an approach to connecting the information and material flows in autonomous cooperating logistics processes. In: Proceedings of the 12th International MITIP Conference on Information Technology \& Innovation Processes of the Enterprises, pp. 54-67 (2010)

Icarte, G.: Negotiations for Agent-based Concurrent Process Scheduling of Resources in Highly Dynamic Environments. Doctoral Dissertation, University of Bremen (2021a)

Icarte, G., Pinto, J.D., Herzog, O.: A dynamic scheduling multiagent system for truck dispatching in open-pit mines. In: Rocha, A.P. et al. (eds.): ICAART 2020, LNAI 12613, pp. 132-148 (2021b)

Jedermann, R., Gehrke, J.D., Becker, M., Behrens, C., Kluge, E.M., Herzog, O., Lang, W.: Transport scenario for the intelligent container. In: Hülsmann, M., Windt, K. (eds.) Understanding Autonomous Cooperation \& Control in Logistics, pp. 393-404. Springer, Berlin (2007)

Kirn, S., Herzog, O., Lockemann, P., Spaniol, O. (eds.): Multiagent Engineering - Theory and Application in Enterprises. Springer, Heidelberg (2006)

Knirsch, P., Timm, I.J.: Adaptive multiagent systems applied on temporal logistics networks. In: Muffatto, M., Pawar, K.S. (eds.): Logistics in the Information Age. Proceedings of the 4th International Symposium on Logistics (ISL-99), Florence, Italy, pp. 213-218 (1999)

Krempels, K.-H., Spaniol, O., Scholz, T., Timm, I.J., Herzog, O.: Interaction design. In: Kirn, S., Herzog, O., Lockemann, P., Spaniol, O. (eds.) Multiagent Engineering - Theory and Application in Enterprises, pp. 383-403. Springer, Heidelberg (2006)

Lang, W., Janßen, S., Jedermann, R.: The intelligent container - a cognitive sensor net for fruit logistics. In: Proceedings of the 3rd International Conference on Sensor Networks (SENSORNETS-2014), pp. 351-359 (2014)

Luhmann, N.: Soziale Systeme. Grundriss einer allgemeinen Theorie. Suhrkamp, Frankfurt, Germany (1984)

Luhmann, N.: Social Systems. Translation of Soziale Systeme. Stanford University Press, Stanford, USA (1995)

Müller, H.-J.: Towards agent systems engineering. Data Knowl. Eng. 23(3), 217-245 (1997)

Multiagent Coordination Enabling Autonomous Logistics. Doctoral Dissertation, University of Bremen (2010)

Poslad, S., Charlton P.: Standardizing agent interoperability: the FIPA approach. In: Luck, M., Mařík, V., Štěpánková, O., Trappl, R. (eds.): Multi-Agent Systems and Applications: 9th ECCAI Advanced Course, ACAI 2001 and Agent Link's 3rd European Agent Systems Summer School, EASSS 2001, Prague, Czech Republic, July 2-13, 2001 Selected Tutorial Papers, pp. 98-117 (2001)

Rao, A.S., Georgeff, M.P.: BDI-agents: from theory to practice. In: Proceedings of the First International Conference on Multiagent Systems, pp. 312-319. AAAI-Press, San Francisco, CA (1995)

Reuter, L., Berndt, J.O., Timm, I.J.: Exploring the effects of role design on agent learning. In: Schmid, U., Klügl, F., Wolter, D. (eds.) KI 2020: Advances in Artificial Intelligence, pp. 190203. Springer, Berlin (2020)

Scholz-Reiter, B., Windt, K., Freitag, M.: Autonomous logistic processes - New demands and first approaches. In: Proceedings of 37th CIRP International Seminar on Manufacturing Systems, pp. 357-362. Computer and Automation Research Institute, Hungarian Academy of Sciences, Budapest (2004) 
Schuldt, A.: Multiagent coordination enabling autonomous logistics. Doctoral Dissertation, University of Bremen, Springer, Heidelberg (2011)

Schumann, R., Lattner, A.D., Timm, I.J.: Management-by-exception - a modern approach to managing self-organizing systems. Commun. SIWN. 4, 168-172 (2008)

Stockheim T., Nimis J., Scholz T., Stehli M.: How to build a multi-multi -agent system - the agent. Enterprise approach. In: Proceedings of the Sixth International Conference on Enterprise Information Systems - Volume 4: ICEIS, pp. 364-371 (2004)

Timm, I.J.: Dynamisches Konfliktmanagement als Verhaltenssteuerung Intelligenter Agenten. Doctoral Dissertation, University of Bremen. DISKI 283. infix: Köln (2004)

Timm, I.J.: Strategic Management of Autonomous Software Systems - Overview Article. TZITechnical Report 35/2006 and part of Habilitation Thesis. University of Bremen: Bremen (2006)

Timm, I.J., Hillebrandt, F.: Reflexion als sozialer Mechanismus zum strategischen Management autonomer Softwaresysteme. In: Schmitt, M., Michael, F., Hillebrandt, F. (eds.) Reflexive soziale Mechanismen. Von soziologischen Erklärungen zu sozionischen Modellen, pp. 255288. VS Verlag, Wiesbaden (2006)

Timm, I.J., Schumann, R.: Performance measurement of multiagent systems: towards dependable MAS. In: Proceedings of the 2009 Spring Simulation Multi Conference ADS, BIS, MSE, MSEng, vol. 41, pp. 177-184. SCS, San Diego, CA (2009)

Timm, I.J., Scholz, T., Herzog, O., Krempels, K.-H., Spaniol, O.: From agents to multiagent systems. In: Kirn, S., Herzog, O., Lockemann, P., Spaniol, O. (eds.) Multiagent Engineering Theory and Application in Enterprises, pp. 35-51. Springer, Heidelberg (2006a)

Timm, I.J., Scholz, T., Fürstenau, H.: From Testing to Theorem Proving. In: Kirn, S., Herzog, O., Lockemann, P., Spaniol, O. (eds.) Multiagent Engineering. International Handbooks on Information Systems. Springer, Berlin, Heidelberg (2006b)

Timm, I. J., Reuter, L., Berndt, J. O., Ulfert, A.-S., Ellwart, T., Antoni, C. H.: Analyzing the effects of role configuration in logistics processes using multiagent-based simulation: An interdisciplinary approach. In: 52nd Hawaii International Conference on System Sciences, HICSS 2019, Maui, Hawaii, USA, January 8-11, pp. 1-10) (2019)

Timm, I. J., Reuter, L., Berndt, J. O.: Role assignment adaptation: an intentional forgetting approach. In: 53rd Hawaii International Conference on System Sciences, HICSS 2020, Maui, Hawaii, USA, January 7-10, pp. 1-10. (2020)

Toenshoff, H.K.: Dynamic modelling of process planning knowledge based on a shop-floor model. In: Proceedings of the 32nd CIRP International Seminar on Manufacturing Systems, pp. 497504. Leuven, Belgium (1999)

Warden, T.: Interactive Multiagent Adaptation of Individual Classification Models for Decision Support. Dissertation, University of Bremen (2019)

Wooldridge, M.J.: Reasoning about Rational Agents. The MIT Press, Cambridge, MA (2000)

Wooldridge, M., Jennings, N.R.: Intelligent agents: theory and practice. Knowl. Eng. Rev. 10(2), $115-152(1995)$

Open Access This chapter is licensed under the terms of the Creative Commons Attribution 4.0 International License (http://creativecommons.org/licenses/by/4.0/), which permits use, sharing, adaptation, distribution and reproduction in any medium or format, as long as you give appropriate credit to the original author(s) and the source, provide a link to the Creative Commons licence and indicate if changes were made.

The images or other third party material in this chapter are included in the chapter's Creative Commons licence, unless indicated otherwise in a credit line to the material. If material is not included in the chapter's Creative Commons licence and your intended use is not permitted by statutory regulation or exceeds the permitted use, you will need to obtain permission directly from the copyright holder.

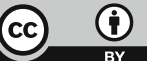

